\title{
Sunspot photometry with phase diversity
}

\section{Methods and global sunspot parameters}

\author{
A. Tritschler and W. Schmidt
}

\author{
Kiepenheuer-Institut für Sonnenphysik, Schöneckstr. 6, 79104 Freiburg, Germany \\ e-mail: ali, wolfgang@kis.uni-freiburg.de
}

Received 8 May 2001 / Accepted 19 November 2001

\begin{abstract}
The global brightness structure of a small sunspot is investigated. Seeing and instrumental effects are compensated by application of the phase-diversity technique and a conventional deconvolution method. We calculate brightness temperatures for the reconstructed data in three simultaneously observed continuum bands of the solar spectrum $(402.1 \mathrm{~nm}, 569.5 \mathrm{~nm}, 709.1 \mathrm{~nm})$. The darkest umbral regions are on average $0.17 I^{\odot}, 0.27 I^{\odot}$ and $0.32 I^{\odot}$ bright. The corresponding temperatures lie in the range around $4790 \mathrm{~K}, 4600 \mathrm{~K}$ and $4460 \mathrm{~K}$. The spatially averaged penumbral brightness amounts to $0.72 I^{\odot}, 0.81 I^{\odot}$ and $0.85 I^{\odot}$, which corresponds to $5910 \mathrm{~K}$, $5750 \mathrm{~K}$ and $5640 \mathrm{~K}$, respectively. Although the spectral distribution of the umbral and penumbral intensities is consistent with former measurements, the derived values support the idea that there exist a real difference in the thermal properties between the umbrae of small and large sunspots.
\end{abstract}

Key words. Sun: sunspots - techniques: image processing

\section{Introduction}

A better understanding of the physics of sunspots goes along with the highest possible spatial resolution because the relevant magnetohydrodynamical processes take place on spatial scales comparable to or even below the pressure scale height and the mean free path length of the photons. In the photosphere this corresponds to distances smaller than $100 \mathrm{~km}$. Theoretical limitations of spatial resolution are given by diffraction effects at the aperture and aberrations of the telescope optics. In practice, the limited spatial resolution is dominated by the influence of the atmospheric turbulence causing fluctuations of the refractive index and temperature of the air, especially in and around the telescope.

Though several techniques have been developed to improve the spatial resolution, there exist only a few scientific applications to solar data. Restricted to speckle and phase-diversity methods, particular interest is devoted to the domain of wavefront sensing and the determination of atmospheric parameters (Acton et al. 1996). On the other hand, investigations mainly focus on properties of either the solar granulation (Druesne et al. 1989; Wilken et al. 1997; Krieg et al. 2000; Hirzberger et al. 2001) or solar magnetic elements in the photosphere (Berger 1997; Berger et al. 1998a,b; van Ballegooijen et al. 1998; Koschinsky et al. 2001). Similar studies for sunspots in

Send offprint requests to: A. Tritschler, e-mail: ali@kis.uni-freiburg.de the context of image reconstruction are limited to the work done by Sütterlin \& Wiehr (1998), who performed a 3-colour photometry on speckle-reconstructed data and subsequently derived temperature maps of a sunspot.

The brightness of sunspots has already been studied observationally in the past. There exist a variety of observations which are only corrected for stray light effects and/or the influence of the telescope based on theoretical assumptions (Maltby 1972; Albregtsen \& Maltby 1981a,b; Grossmann-Doerth \& Schmidt 1981; Albregtsen et al. 1984; van Ballegooijen 1984; Sobotka 1988; Bumba et al. 1990). All these studies are restricted to rather large sunspots, where the stray light problem can be mastered appropriately. Addressed are questions like whether and how the emitted sunspot radiation depends on parameters like wavelength, size, magnetic field strength, filling with umbral dots (UDs), position and the solar cycle. Thus for large umbrae the effective temperature is typically about $3700 \mathrm{~K}$. In the wavelength region between $387 \mathrm{~nm}$ and $2.35 \mu \mathrm{m}$ the continuum intensity of umbral cores increases from $0.01 I^{\odot}$ to $0.57 I^{\odot}$, whereas the spatially averaged penumbra shows intensities in the range from $0.64 I^{\odot}$ to $0.93 I^{\odot}$. For further details, we refer to the extensive reviews given by Maltby (1992) and Solanki (1997).

The contents of this article (Paper I) include the specification about the data reduction, applied image reconstruction techniques and the determination 


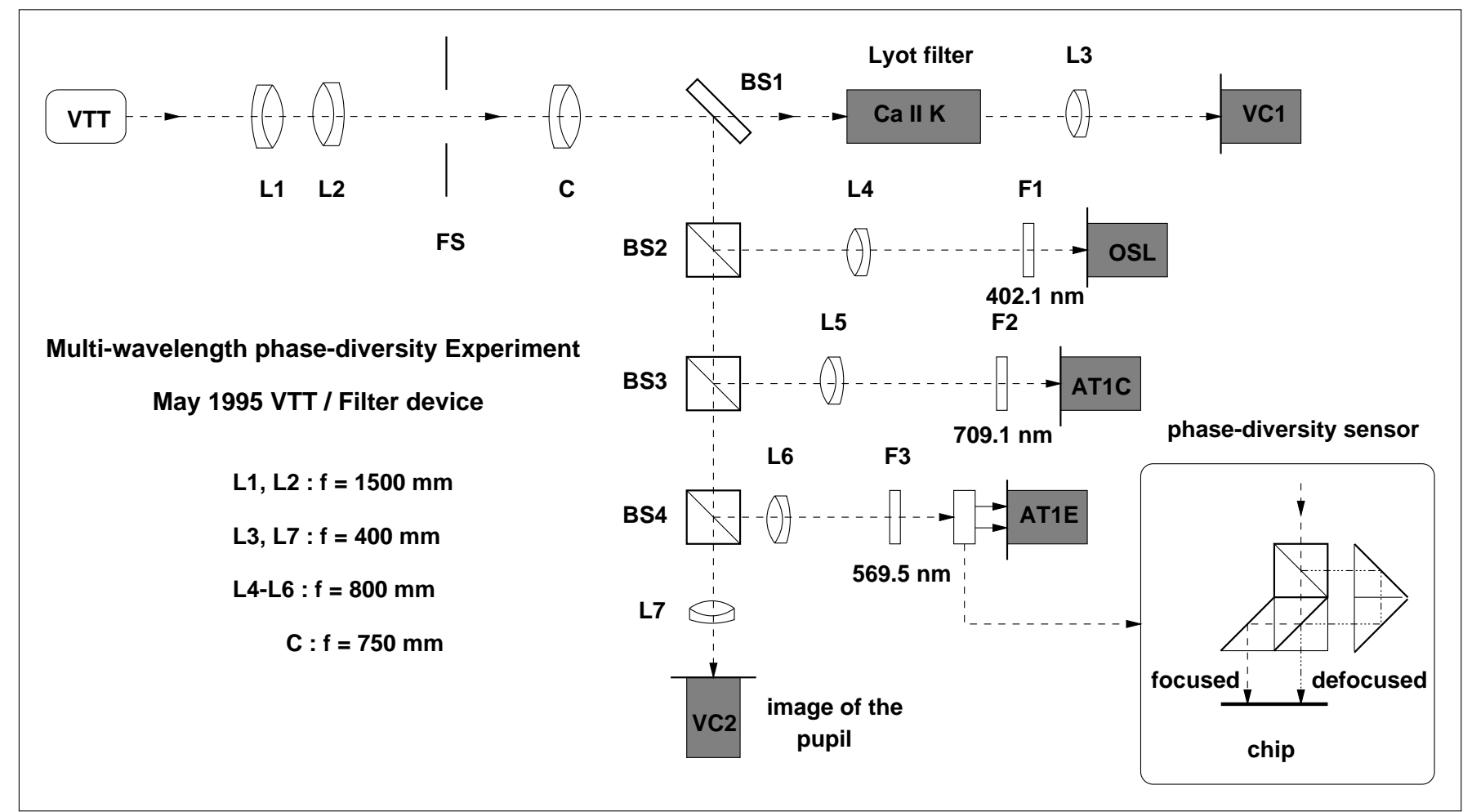

Fig. 1. Optical setup of the phase-diversity experiment. VC: video camera, BS: beam splitter, F: interference filter, FS: field stop, L: imaging lens, C: collimator lens. PS: pupil iris.

of typical sunspot properties like umbral and penumbral brightness and the corresponding brightness temperatures. In a forthcoming article (Paper II) the investigation will be expanded to morphological and brightness characteristics of the sunspot fine structure.

\section{Observations}

Observations were made on May 19, 1995, with the German Vacuum Tower Telescope (VTT) located at the Observatorio del Teide on Tenerife. The VTT is a $70 \mathrm{~cm}$ $\mathrm{f} / 46$ reflector telescope in a vacuum tower configuration with a prime-focus image scale of $4.6 \operatorname{arcsec} \mathrm{mm}^{-1}$. In order to observe simultaneously in three different continuum bands of the solar spectrum, the observations employed the Multichannel Filter System of the VTT (Kentischer 1995). The optical setup is shown schematically in Fig. 1. Three Andover manufactured interference filters centered at $402.1 \mathrm{~nm}, 569.5 \mathrm{~nm}$ and $709.1 \mathrm{~nm}$ with full width at half maximum (FWHM) bandpasses of $1 \mathrm{~nm}, 3 \mathrm{~nm}$ and $0.4 \mathrm{~nm}$, respectively, are placed directly in front of three large format $(1024 \times 1024)$ CCD cameras recording the data. The Peltier-cooled CCDs (AT1E, AT1C) have a pixel size of $19 \mu \mathrm{m}$ and 16 bit readout. The third CCD camera (OSL) has 12 bit readout with $18.6 \mu \mathrm{m}$ pixel size. A MicroVax was used to control the OSL and to trigger the other cameras. A circular field stop FS $(7.84 \mathrm{~mm}$ in diameter) gave a field of view (FOV) with a nominal diameter of $\sim 36$ arcsec. Together with the assumed magnification of the filter device (resulting from the focal length ratio of camera lens to collimator lens, 800:750), the image scale of the AT-cameras $(569.5 \mathrm{~nm}, 709.1 \mathrm{~nm})$ results in 0.0819 arcsec pixel $^{-1}$ whereas the smaller pixel size of the OSL-camera $(402.1 \mathrm{~nm})$ lead to an image scale of 0.0802 arcsec pixel $^{-1}$. The exposure time was $48 \mathrm{~ms}$ at the beginning and was later reduced to $38 \mathrm{~ms}$. The cycle time was about $15 \mathrm{~s}$.

In order to apply the phase-diversity technique, a defocused image was taken additionally in one of the wavelength channels ( $569.5 \mathrm{~nm}$, phase-diversity channel). The amount of defocusing was $22.37 \mathrm{~mm}$ for $569.5 \mathrm{~nm}$, corresponding to a phase shift of 1 wave at the edge of the aperture. The use of a beam splitter device which is fixed in front of the CCD camera allows to image both the focused and the defocused image onto one CCD chip. This reduces the number of cameras needed, simplifies the alignment of the optics and guarantees the strict simultaneity between the focused and defocused frame. However, the use of such a PD assembly restricts the beam size and therefore the total FOV.

In order to derive the actual magnification of the filter device for each of the wavelength channels, resolution target frames were taken. Therefore a calibrated target slide was inserted at the prime focus FS of the VTT and reimaged to the three CCDs. The target frames are also used to estimate the differential aberrations between the channels (Sect. 3). In addition, exposures of the solar limb in each orientation were obtained and used to estimate the amount of stray light in the data. 

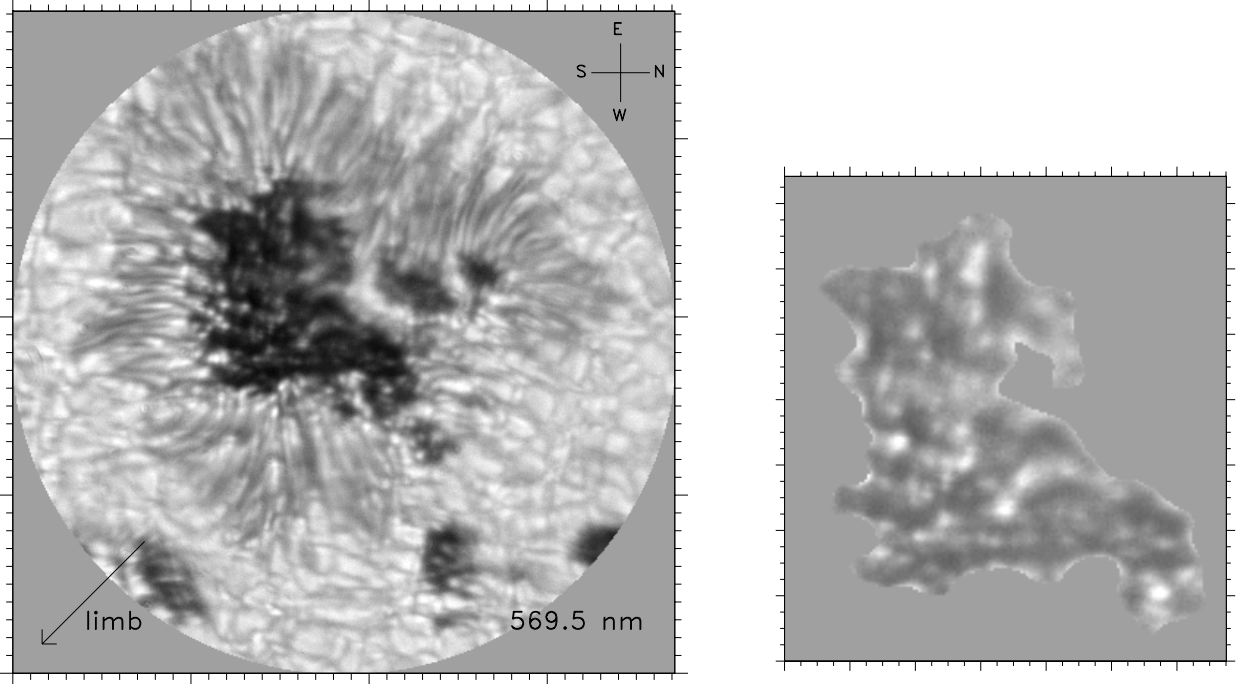

Fig. 2. The observed sunspot taken at 8:18 UT in the continuum near $569.5 \mathrm{~nm}$. Left: total FOV (scale: 1 tick mark $\widehat{=} 1$ arcsec). Right: umbra (scale: 1 tick mark $\widehat{=} 0.5$ arcsec).

The analyzed data set covers a $\sim 90$ min sequence of a well developed sunspot (f-spot, 25 arcsec in diameter), part of a bipolar region (NOAA 7871), observed out of disc center $(\mu=0.79)$. Figure 2 illustrates the variety of structures by means of a typical exposure in the continuum near $569.5 \mathrm{~nm}$.

\section{Data reduction}

The first steps of the analysis included gain correction and subtraction of dark current. Several dark images were taken by shutting off the path of direct sunlight to the detectors. Flatfield images were generated by moving the focused telescope during the exposure. In this way about 50 frames were taken. We selected those with lowest intensity-rms and averaged them in order to erase any residual solar structures in the images.

Due to the different pixel sizes of the CCD cameras and the differing optical elements in the three wavelength channels, we had to rescale and shift the images and to correct for image rotation. Image rotation mainly results from small differences in the orientation of the CCD cameras. The actual magnification of the filter device and the rotation angle of the images with respect to the green reference were derived from evaluation of the calibrated target frames. The rescaling was done by Fourier resampling. As a consequence of the difference between the nominal and actual magnification, the defocus was only 0.863 waves instead of 1 wave. Rotation of the blue $(402.1 \mathrm{~nm})$ and the red $(709.1 \mathrm{~nm})$ channel against the PD-channel amounts to 0.60 and -0.25 degrees respectively, whereas rotation between the focused and the defocused image was negligible $(<0.1$ degrees $)$.

Close inspection and comparison of the target frames in the three continua results for the blue, green and red wavelength channel in a spatial resolution of $22.62 \mathrm{LP} \mathrm{mm}^{-1}, 20.16 \mathrm{LP} \mathrm{mm}^{-1}$ and $17.96 \mathrm{LP} \mathrm{mm}^{-1}$ corresponding to 0.203 arcsec, 0.228 arcsec and 0.256 arcsec.
Therefore, the spatial resolution achieved in the data compared to the corresponding diffraction limit is poorer for the blue continuum than for the red continuum. Besides, the targets show a slight astigmatism, visible as a difference in resolution between horizontal and vertical bars in the target frames. The difference amounts to $\sim 2 \mathrm{LP} \mathrm{mm}^{-1}$ $(\sim 0.03$ arcsec) independent from wavelength. We cannot make out any other differential aberrations and therefore consider them as small enough to be negligible.

Image alignment of the whole sequence with respect to the reference channel $(569.5 \mathrm{~nm})$ was done with subpixel accuracy. For shifts between the focused and defocused frames, pixel accuracy is sufficient, since residual shifts are compensated for by the image-reconstruction algorithm. From the full data set we selected those images with highest granular rms-contrast and lowest intensity ratio umbra to mean quiet sun. Further visual inspection and selection resulted in 71 exposures with a mean time lag of $1.2 \mathrm{~min}$ (maximum time lag 6.2 min) used for further investigation.

\section{Image restoration}

\subsection{Phase-diversity}

Effectively removing the distortions introduced by the telescope/atmosphere combination requires the determination or estimation of the actual wavefront. The phasediversity technique is comparable to a focal volume technique, in the sense, that the information about the phase aberrations is extracted by analyzing the intensity inside and outside the focal plane. In principle, the method makes use of the combined information contained in two images: the focused image and an intentionally defocused one. This image pair contains sufficient information to estimate the true intensity as well as the phase aberrations. The principles of the conventional phase-diversity technique are described in detail by Gonsalves (1982) 
and Löfdahl \& Scharmer (1994). Further developed methods like the Joint and Partitioned Phase-Diverse Speckle technique (JPDS and PPDS respectively) are found in Paxman et al. (1992, 1996). A review of all existing phasediversity techniques and further references are given by Löfdahl (1996).

\subsection{Wavefront scaling and deconvolution}

The phase-diversity algorithm yields an estimate of the phase aberration prameters $\alpha_{\mathrm{j}}$, which are used to form the OTF for the focused $\left(T_{1}\right)$ and the defocused image $\left(T_{2}\right)$. In principle the resulting focused OTF can be subsequently used to restore the other wavelength channels via a deconvolution process:

$f=\mathcal{F}\left\{\frac{D}{T_{1}}\right\}$

where $D$ is the Fourier transformed image at the additional wavelength. Since the PSF - and consequently the OTF - are not wavelength independent, directly using the PD-OTF for the deconvolution leads to an under- and overcorrection in the blue and the red spectral region respectively. Therefore a wavelength dependent scaling of the phase function becomes necessary.

We consider the phase function composed of contributions assigned to seeing and the influence of the combined triple system telescope - filter device - PD assembly. Since the instrumental effects are regarded as constant in time during the $90 \mathrm{~min}$ observations, the phase function splits in two parts: a time dependent component $\phi_{\mathrm{s}}(\lambda, \boldsymbol{x}, t)$ originating from seeing and a time independent instrumental component $\phi_{\mathrm{i}}(\lambda, \boldsymbol{x})$ :

$\phi(\lambda, \boldsymbol{x}, t)=\phi_{\mathrm{s}}(\lambda, \boldsymbol{x}, t)+\phi_{\mathrm{i}}(\lambda, \boldsymbol{x})$.

The vector $\boldsymbol{x}$ denotes the two-dimensional coordinate in the pupil plane. The wavelength dependency of the individual contributions is unknown, but because of the PDtechnique the phase function is estimated for a reference wavelength $\phi\left(\lambda_{\mathrm{r}}, \boldsymbol{x}, t\right)$ with $\lambda_{\mathrm{r}}=569.5 \mathrm{~nm}$. In the following it is assumed that the wavelength dependency of the phase function can be modelled by a simple scaling of the reference phase

$\phi(\lambda, \boldsymbol{x}, t) \approx C_{\mathrm{s}}\left(\lambda, \lambda_{\mathrm{r}}\right) \phi_{\mathrm{s}}\left(\lambda_{\mathrm{r}}, \boldsymbol{x}, t\right)+C_{\mathrm{i}}\left(\lambda, \lambda_{\mathrm{r}}\right) \phi_{\mathrm{i}}\left(\lambda_{\mathrm{r}}, \boldsymbol{x}\right)$.

As a starting point for the following considerations, we make use of the relationship

$l_{\mathrm{opt}}(\boldsymbol{x}, \lambda)=\int_{0}^{L} n\left(\boldsymbol{x}, s^{\prime}, \lambda\right) \mathrm{d} s^{\prime}$

between the optical path length $l_{\text {opt }}$ and the refractive index $n\left(\boldsymbol{x}, s^{\prime}, \lambda\right)$ of a medium.

The image-forming optics of the telescope is based on reflective surfaces. Thus only the influence of glas elements (entrance window, beam splitters, lenses) in the light path is important for the estimation of $C_{\mathrm{i}}$. An originally plane wave, which passes through glas, deforms to a spherical wave centered at the focal plane. Though for achromatic elements of effective thickness $L$, the optical path length $l_{\text {opt }}$ is independent of wavelength, but the corresponding phase

$\phi(\lambda, \boldsymbol{x})=\frac{2 \pi l_{\mathrm{opt}}(\boldsymbol{x})}{\lambda}$.

is not. The $C_{\mathrm{i}}$ is now derived from

$\frac{\phi_{\mathrm{i}}(\lambda, \boldsymbol{x})}{\phi_{\mathrm{i}}\left(\lambda_{\mathrm{r}}, \boldsymbol{x}\right)}=\frac{\lambda_{\mathrm{r}}}{\lambda}=C_{\mathrm{i}}\left(\lambda, \lambda_{\mathrm{r}}\right)$

and amounts to

$C_{\mathrm{i}}\left(\lambda, \lambda_{\mathrm{r}}\right)= \begin{cases}1.416: & 402.1 \mathrm{~nm} \\ 0.803: & 709.1 \mathrm{~nm} .\end{cases}$

Concerning the parameter $C_{\mathrm{s}}$, the original wavefront from the sun is regarded as plane outside the earth's atmosphere and subsequently gets distorted due to the random distributed fluctuations of the refractive index. Since neither the height dependence of the refractive index nor its temporal behaviour is known, the following simplification is used in order to derive a plausible functional form of the parameter $C_{\mathrm{s}}$. Imagine the plane wavefront encounters an air bubble with refractive index $n$ and height $L$ surrounded by vacuum. Some part of the wavefront will travel through the bubble, while the rest propagates unperturbed. In the following we take the external wavefront as a reference. The optical pathlength $l_{\text {opt }}$ is related to the refractive index via Eq. (4). We neglect any height dependence and horizontal variations of $n$ and obtain

$l_{\text {opt }}(\lambda)=n(\lambda) L$

Thus, while the outer wavefront just travels the distance $L$, the internal wavefront travels the distance $n(\lambda) L$. Hence the difference is given by

$\Delta l_{\text {opt }}(\lambda)=(n(\lambda)-1) L$

The differences in the optical pathlengths $\Delta l_{\text {opt }}$ can be recalculated in phase differences via Eq. (5)

$C_{\mathrm{s}}(\lambda)=\frac{\lambda_{\mathrm{r}}}{\lambda} \frac{(n-1)}{\left(n_{\mathrm{r}}-1\right)}$

for the seeing parameter with values

$C_{\mathrm{s}}\left(\lambda, \lambda_{\mathrm{r}}\right)= \begin{cases}1.441: & 402.1 \mathrm{~nm} \\ 0.797: & 709.1 \mathrm{~nm}\end{cases}$

The wavelength dependence of the refractive index $n(\lambda)$ is taken from Allen (1955). In a final step the corresponding aberration function $\phi$, the proper pupil transmission function and finally the OTFs are recalculated. 


\section{Image processing}

Our phase-diversity algorithm is based mainly on the approach of Löfdahl \& Scharmer (1994) and a modified version of its IDL implementation by Acton (priv. comm.). This version treats the defocus as a fixed parameter and works with a single image pair to create one restored scene. The use of only one image pair can lead to zeroes in the OTF. At these frequencies, all information about the object is lost. In order to avoid this effect, we selected only those images representative for the best seeing conditions in our sequence.

For all reconstructions we used the first 15 Zernike polynomials described by Noll (1976) to parametrize the phase aberration function $\phi$. Because phase-diversity algorithms are insensitive to measure the piston- and tilt terms, the first three coefficients in the polynomial expansion of $\phi$ are set to zero. We also tested the algorithm with the use of $6,10,21$ and 45 Zernike polynomials for the representation of the phase function. For more than 15 Zernike polynomials, this resulted in no substantial improvement of the reconstructions, the results for 15 Zernike and 21 Zernike are hard to distinguish.

In order to avoid unlimited amplification of noise, a modified optimum filter is applied to the data in each iteration step (see Löfdahl \& Scharmer 1994 for a detailed description). This filter depends on the instantaneous phaseaberration parameters, derived by the algorithm, so that the filter itself changes from iteration to iteration. Prior to deconvolving the other wavelengths, the image data is filtered by applying an optimum filter. For the generation of the filter, the mean noise power spectrum of the data is estimated from the average power spectrum of the flatfield images. Since there is substantial power in the latter at low frequencies due to residual solar structure, the mean noise power was calculated from the high frequency tail of the power spectrum by averaging over all frequencies above the cutoff-frequency of the telescope.

In the next steps we considered the influence of anisoplanatism and the consequences of apodization. Light propagation through the earth's atmosphere is strongly anisoplanatic. This results in a spatial variation of image sharpness over the FOV, leading to the so-called isoplanatic patches. Therefore, the whole FOV is divided into overlapping subfields, which are reconstructed individually and subsequently patched together (von der Lühe 1993).

The data are multiplied by a suitable window function in order to avoid spurious high frequency patterns in the Fourier domain that produce large edge effects in the image space.

The chosen FOV is $318 \times 318$ pixel $\left(\sim 26 \times 26 \operatorname{arcsec}^{2}\right)$, divided into 25 subfields of size $128 \times 128$ pixel $(10.5 \times$ $10.5 \operatorname{arcsec}^{2}$ ) with $70 \%$ overlap. For the estimation of the phase aberration parameters, the inner $70 \times 70$ pixel of each subfield are used. A modified Hanning window apodizes the outer 16 pixel of the subfield but leaves the central region unchanged. Noise filtering is applied until the third

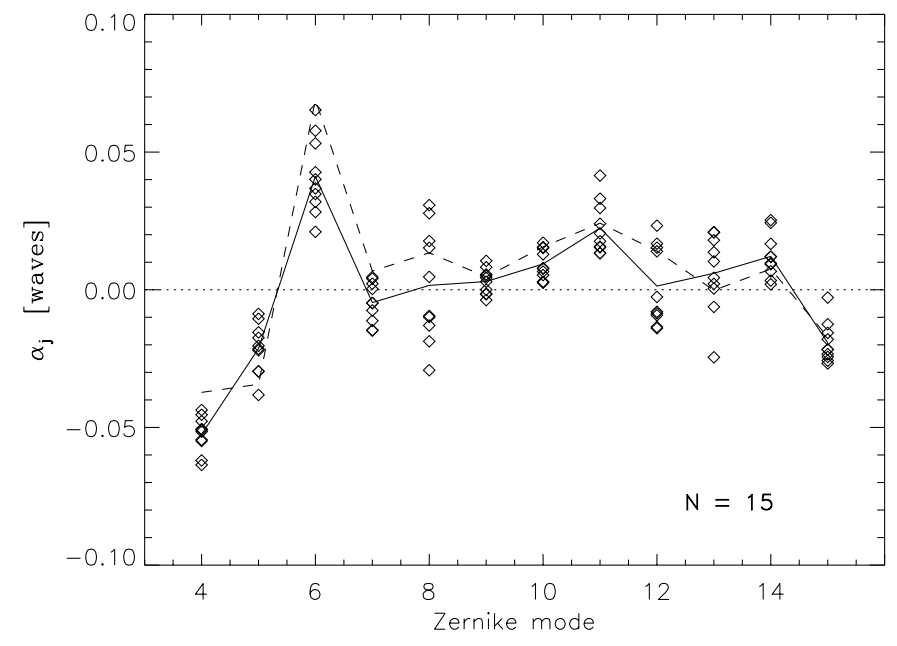

Fig. 3. Averaged Zernike coefficients derived from 10 nonoverlapping subfields ( $\diamond$-symbols). The average over the individual subfields is indicated by the full line, whereas the dashed line shows the result obtained from the restoration of averaged image pairs (see text). The Zernike polynomials are normalized after Noll (1976).

iteration step. The maximum (minimum) number of iterations is limited to $10(3)$.

\subsection{Fixed aberrations}

The Zernike coefficients are averaged in time to determine the fixed aberrations of the combined triple system telescope - filter device - PD assembly (Acton et al. 1996). Figure 3 shows the result. Displayed are the averaged Zernike coefficients $\alpha_{4}-\alpha_{15}$ derived from 10 nonoverlapping subfields. The symbols denote the result for the individual subfields, whereas the full line specifies the mean value, corresponding to the field-independent average aberration.

Alternatively, all subfield images can be averaged in time to produce a single image pair focused/defocused. Restoration of the scene from this average image pair, as proposed by Löfdahl \& Scharmer (1994), yields directly the fixed aberrations, presuming that the average is taken over a time scale smaller than the dynamical time scale of the solar feature under consideration. The observation period of $90 \mathrm{~min}$ makes this method rather inadequate for our data set. However, the result is included in Fig. 3 and indicated by the dashed line.

Knowledge of the fixed aberrations allows to give a rough estimate of the Fried parameter $r_{0}$. This is done by calculation of the variances of the individual Zernike coefficients after subtraction of the contribution from the fixed aberrations. Presuming that the power spectrum of the refractive index fluctuations is of Kolmogorov type, a correlation model for the phase function is given and the variances can be theoretically calculated in dependence from $r_{0}$ and the aperture $D$. Comparison with the observed variances leads to a Fried parameter $r_{0}$ of approximately $25 \mathrm{~cm}$ at $569.5 \mathrm{~nm}$. 
Table 1. List of semi-empirical model atmospheres used for the calculation of absolute intensities. The first column denotes the abbreviations used in the text.

\begin{tabular}{lll}
\hline QS & Quiet sun & Holweger \& Müller (1974) \\
KM & Penumbra & Kjeldseth Moe \& Maltby (1969) \\
DF & Penumbra & Ding \& Fang (1989) \\
OM & Umbra (cold) & Obridko \& Staude (1988) \\
OS & Umbra (hot) & \\
M & Umbra (L) & Maltby et al. (1986) \\
HOT & Umbra (small) & Collados et al. (1994) \\
COOL & Umbra (big) & \\
M4 & Umbra & Kollatschny et al. (1980) \\
\hline
\end{tabular}

\section{Determination of temperatures}

The calibration is done with the intensity emitted by the quiet sun in the continuum of the observed wavelength bands. In a first step, the measured photons must be set into relation to the intensity. This presumes knowledge of the spectral intensity distribution, $I_{\lambda}$, and its centerto-limb variation, since our data is not obtained at disk center. We used the disk-center intensities measured by Neckel \& Labs (1984) and the limb darkening given by Neckel (1996) in order to derive the intensities $I_{\lambda}(\mu)$ for our three observed continuum wavelengths. In a second step, each image was multiplied by the intensity ratio $I_{\lambda}(\mu) / I_{\lambda}^{\odot}$, where $I_{\lambda}^{\odot}$ denotes the observed average intensity of the quiet sun.

In principle, the intensities $I_{\lambda}(\mu)$ can be inferred by means of a numerical code solving for the radiation transfer equation for the intensity. This was done additionally, but rather to compare the measured intensities and derived temperatures with the synthetic ones. The numerical calculations have been done with a $1 \mathrm{~d}$ radiation transfer code on the basis of several model atmospheres, reflecting the different thermodynamic conditions in and outside the spot's photosphere. For a summary of the applied models, see Table 1.

\subsection{Brightness and colour temperatures}

For the interpretation of the continuum intensities we assume LTE throughout the solar photosphere and use the Planck law to convert the intensities into brightness temperatures $T_{\mathrm{B}}$. Since the continuum opacities are wavelength dependent, these temperatures are wavelength dependent as well; they provide a measure of the temperature and its gradients at the continuum formation height, i.e., the deep photosphere. Due to the presence of scattering, however, a direct identification of brightness temperatures with actual temperatures in the atmosphere is not possible without detailed modelling. In addition, towards shorter wavelengths the density of absorption lines is so large that the brightness temperatures are significantly lower than the temperatures in the continuum formation region. Alternatively, colour temperatures $T_{\mathrm{C}}$ can be

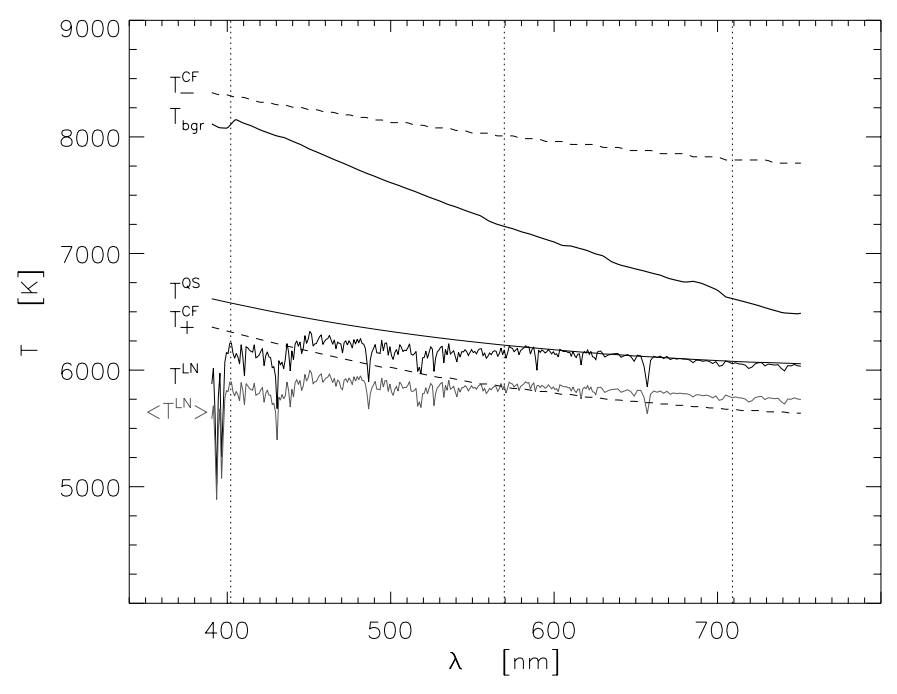

Fig. 4. Spectral distribution of brightness and colour temperatures (disk center). $T_{\mathrm{bgr}}$ : colour temperature based on the wavelengths $(b=\lambda-\Delta \lambda, g=\lambda, r=\lambda+\Delta \lambda)(\Delta \lambda=25 \mathrm{~nm}) . T^{\mathrm{QS}}$ : brightness temperature from $I^{\mathrm{QS}}$ (Holweger-Müller model QS). $I^{\mathrm{LN}}$ : intensity from Labs \& Neckel (1984). $T^{\mathrm{LN}}$ : brightness temperature from $I^{\mathrm{LN}} .\left\langle T^{\mathrm{LN}}\right\rangle$ : brightness temperature from $\left\langle I^{\mathrm{LN}}\right\rangle$. $T_{-}^{\mathrm{CF}}$ : temperature at $\log \tau_{-} . T_{+}^{\mathrm{CF}}$ : temperature at $\log \tau_{+}$.

considered. Unlike the brightness temperature $T_{\mathrm{B}}$, which is defined by the intensity at one specific wavelength in the corresponding Planck spectrum, the colour temperature results from the intensity ratio at two different wavelength points:

$\frac{I_{\lambda_{1}}}{I_{\lambda_{2}}}=\frac{B_{\lambda_{1}}\left(T_{\mathrm{C}}\right)}{B_{\lambda_{2}}\left(T_{\mathrm{C}}\right)} \quad T_{\mathrm{C}}=T_{\mathrm{C}}\left(\lambda_{1}, \lambda_{2}\right)$.

This can be extended to the definition of multi-colour temperatures. For the observed (Neckel \& Labs 1984) and the synthetic (Holweger-Müller QS model) solar continuum intensity distribution, the spectral curves of $T_{\mathrm{B}}$ and $T_{\mathrm{C}}$ are investigated. Figure 4 shows the corresponding curves. $T_{\mathrm{bgr}}$ denotes the three-colour temperature based on the synthetic continuum intensity distribution in the wavelength interval $(b=\lambda-\Delta \lambda, g=\lambda, r=\lambda+\Delta \lambda)$ with a $\Delta \lambda=25 \mathrm{~nm}$. All other curves represent brightness temperatures $T_{\mathrm{B}}$ and refer to $\mu=1$ except for $\left\langle T^{\mathrm{LN}}\right\rangle$, which is based on the average spectrum of Neckel \& Labs (1984). The temperatures $T_{-}^{\mathrm{CF}}$ and $T_{+}^{\mathrm{CF}}$ refer to the temperatures at the lower and upper limit, resp., of the continuum forming layers, defined by the heights where the contribution function $\mathrm{CF}$ of the emerging continuum intensity drops to $1 / 3$ of its peak value. In this sense $T_{-}^{\mathrm{CF}}$ corresponds to deeper and therefore hotter layers.

The theoretical brightness temperatures show a rather smooth variation with wavelength. The observed distributions $T^{\mathrm{LN}}$ und $<T^{\mathrm{LN}}>$ differ from the (synthetic) continuum curve $T^{Q S}$ particularly for wavelengths $<450 \mathrm{~nm}$ due to the influence of the large number of absorption lines. $T_{\text {bgr }}$ varies strongly with wavelength. In our observed continuum bands $(402.1 \mathrm{~nm}, 569.5 \mathrm{~nm}, 709.1 \mathrm{~nm})$ the difference to $T^{\mathrm{QS}}$ is about $1500 \mathrm{~K}, 1000 \mathrm{~K}$ and $560 \mathrm{~K}$, 
respectively. The behaviour of the colour temperature is a natural consequence of the decrease of $T^{\mathrm{QS}}$ with wavelength. The intensity gradient, $\partial I / \partial \lambda$, derived from the wavelength interval $\left(\lambda_{\mathrm{g}}, \lambda_{\mathrm{r}}\right)$,

$\frac{\partial I}{\partial \lambda} \approx \frac{I_{\mathrm{r}}-I_{\mathrm{g}}}{\lambda_{\mathrm{r}}-\lambda_{\mathrm{g}}}=\frac{B_{\mathrm{r}}\left(T_{\mathrm{r}}\right)-B_{\mathrm{g}}\left(T_{\mathrm{g}}\right)}{\lambda_{\mathrm{r}}-\lambda_{\mathrm{g}}}$,

is steeper than that given by any black body of temperature $T \in\left[T_{\mathrm{r}}, T_{\mathrm{g}}\right]$ :

$$
\left|\frac{\partial I}{\partial \lambda}\right|>\left|\frac{\partial B}{\partial \lambda}\right|_{T} \mid \text {. }
$$

Therefore, $\partial I / \partial \lambda$ can be modelled only with temperatures $T>T_{\mathrm{g}}$. Thus colour temperatures $T_{\mathrm{gr}}$ are higher than the corresponding brightness temperatures $T_{\mathrm{g}}$ and $T_{\mathrm{r}}$. For a perfect black body, Eq. (14) would be an equality. Towards shorter wavelengths, the gradient $\partial B / \partial \lambda$ steepens first, but then decreases because the maximum of the Planck curve is approached. Thus, towards shorter wavelengths higher colour temperatures are needed to model a nonzero value of the intensity gradient $\partial I / \partial \lambda$. This explains the wavelength dependence of $T_{\mathrm{bgr}}$ in Fig. 4.

\section{Error sources}

\subsection{Photospheric stray light}

The sunspot filtergrams are, according to their wavelengths, differently affected by stray light scattered into the umbra by scattering processes in the earth's atmosphere. We used the limb data taken in each wavelength to estimate the amount of stray light. In each observed wavelength band and at each orientation of the solar limb $(\mathrm{N} / \mathrm{W} / \mathrm{E} / \mathrm{S})$ spatially averaged intensity profiles of the solar limb $(\sim 20$ arcsec $)$ were determined. The solar limb was defined as the location of the inflection point of the profile. For different distances to the limb, the intensity ratio of remaining intensity at the particular distance to maximal intensity in the limb profile was calculated and considered as stray light. As expected, we find a decrease of stray light with increasing wavelength. At a limb distance of 5 arcsec (which corresponds roughly to the umbral radius of the observed spot) the stray light amounts to $4-6 \%$, $2-3 \%$ and $1.5-2.5 \%$ for $402.1 \mathrm{~nm}, 569.5 \mathrm{~nm}$ and $709.1 \mathrm{~nm}$ respectively.

\subsection{Estimation of the quiet sun}

In the context of intensity calibration the question arises whether the observed spot surroundings are representative for the undisturbed solar photosphere. How is the brightness and the morphological structure of the granular pattern influenced by the presence of an enhanced magnetic flux? The theory of interaction between convective motions and the magnetic field predicts a smaller cell size if the fluid is permeated by a magnetic field (Chandrasekhar 1961). Investigations of this effect include the study of the solar granulation in coexistence with enhanced magnetic field: active regions, plages and in the direct vicinity of sunspots (e.g. Title et al. 1987; Schmidt et al. 1988; Keller \& Koutchmy 1991; Title et al. 1992). In summary, we assume that the small granular region that is available for the calibration sufficiently represents the quiet sun and that a misjudgement of the average quiet sun intensity does not exceed 1\%. The consequence of a potential error in intensity and its effect on temperature can be estimated via the law of error propagation applied to Planck's law. For typical temperatures in the range $4000-6500 \mathrm{~K}$ this leads to an inaccuracy of $<20 \mathrm{~K}$.

\section{Photometry of the observed sunspot}

The umbra (penumbra) in each image is determined by an individual intensity threshold in the corresponding smoothed version (boxcar average of size $0.74 \times$ $0.74 \operatorname{arcsec}^{2}$ ) of the wavelength-averaged frame. The umbra is defined by $1 / 3$ of the difference between the mean spot surroundings, $I^{\odot}$, and the umbral minimum, $I_{\min }^{\mathrm{u}}$, in the averaged frame. For the definition of the penumbra two values are necessary. The upper limit is set to $0.93 I^{\odot}$, the lower limit to $1 / 2$ of the difference between $I^{\odot}$ and $I_{\text {min }}^{\mathrm{u}}$ in the averaged frame. By this definition, the penumbra is separated from the umbra by a boundary region. The so defined masks are applied to the individual continuum intensity and temperature maps. An example of what is defined as "umbra" is shown on the right hand side of Fig. 2.

In the following, the properties of the overall structure of the observed sunspot are described. We investigate the wavelength dependency of the relative intensities (in units of the mean quiet sun $I^{\odot}$ ) and brightness temperatures. Of special interest are both the minimum (maximum) brightness of the umbra (penumbra), given by $I_{\min }^{\mathrm{u}}\left(I_{\max }^{\mathrm{p}}\right)$, and the spatial averages of umbral and penumbral brightness $<I^{\mathrm{u}}>$ and $\left\langle I^{\mathrm{p}}>\right.$ as well as the relative penumbral intensity fluctuations $C_{\mathrm{rms}}^{\mathrm{p}}$. Spatial and temporal averages are indicated by squared brackets and bars respectively. For reasons of clarity and readability the representation of the results is restricted to the green spectral range $(569.5 \mathrm{~nm})$. The corresponding information about the other two wavelength bands can be extracted from the figures and Table 3 at the end of Sect. 9. The stray light correction is applied only to the darkest part of the umbra, where we subtract an estimated stray light contribution of $6 \%, 3 \%$ and $2.5 \%$ for $402.1 \mathrm{~nm}, 569.5 \mathrm{~nm}$ and $709.1 \mathrm{~nm}$, respectively. Penumbral intensities and spatial brightness averages are given without correction.

\subsection{Observed intensities and temperatures}

The mean brightness of the total umbra is about $0.42 I^{\odot}$ corresponding to $5000 \mathrm{~K}$. This is $\sim 1000 \mathrm{~K}$ less than the mean temperature of the spot surroundings $(6040 \mathrm{~K})$. The spatially averaged penumbral brightness amounts to $0.81 I^{\odot}(5750 \mathrm{~K})$ which is on average twice as bright as the whole umbra. Temporal fluctuations of the spatial 

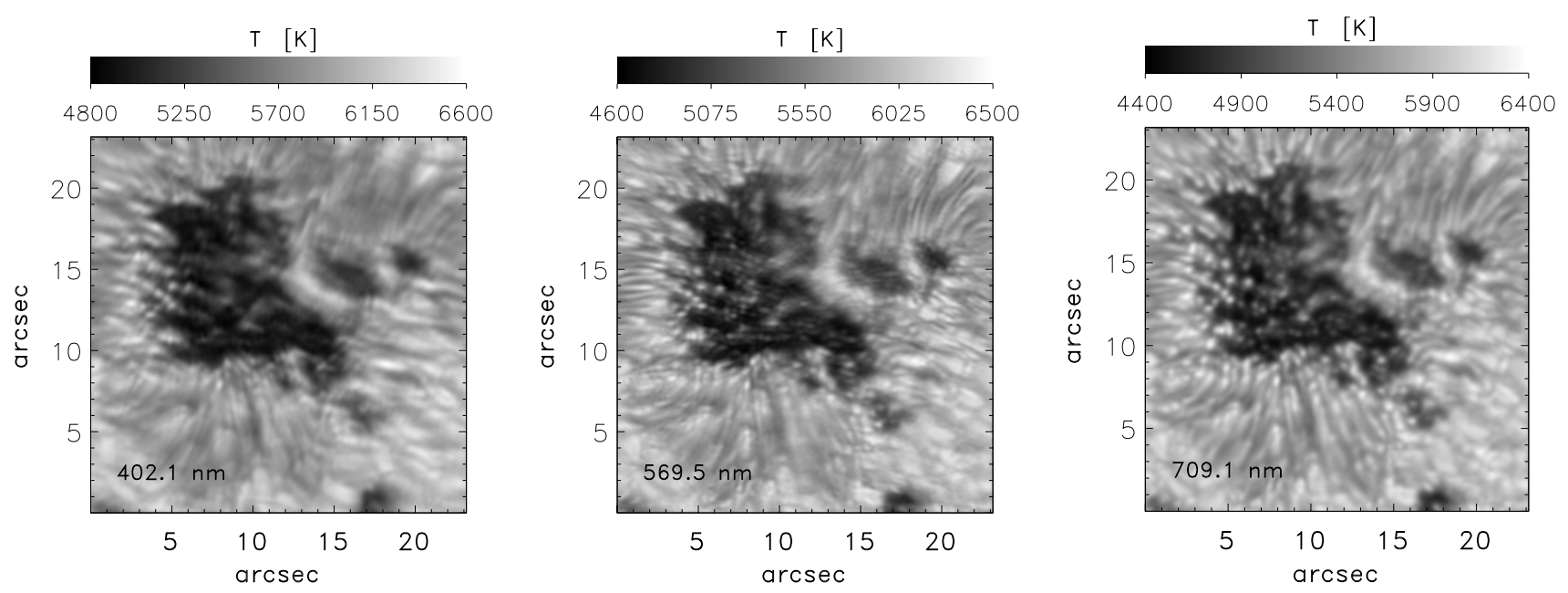

Fig. 5. Typical temperature maps based on the phase-diversity reconstructed intensities (569.5 nm) and the deconvolved intensities $(402.1 \mathrm{~nm}, 709.1 \mathrm{~nm})$.
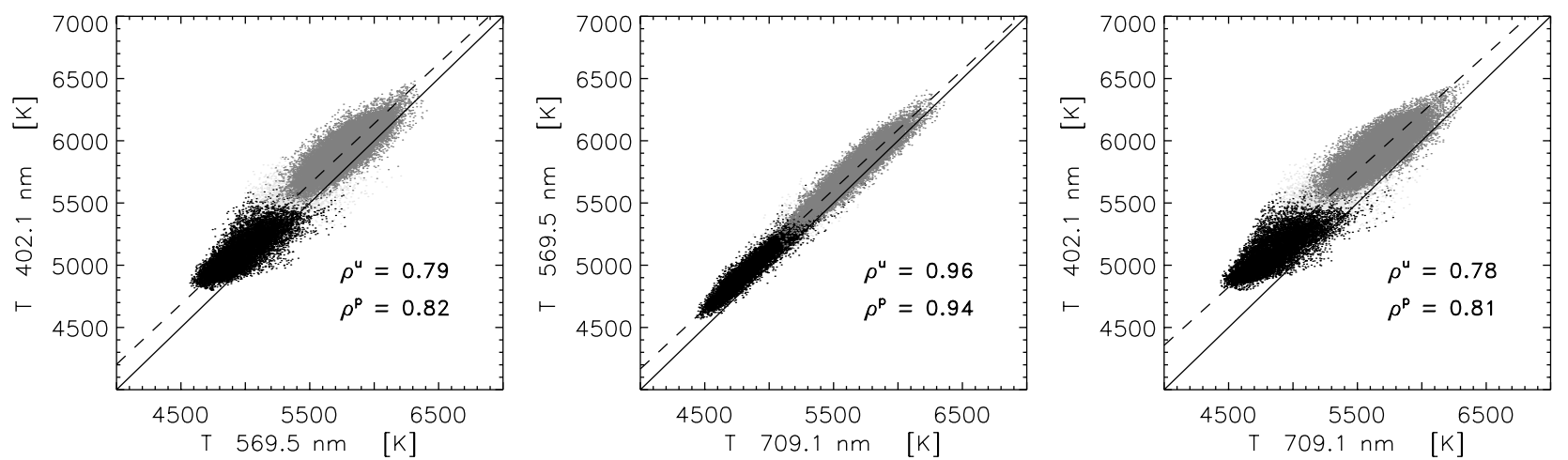

Fig. 6. Correlation of brightness temperatures in the umbra (black points) and penumbra (grey points) based on the reconstructed data set. $\rho$ : linear correlation coefficient of the corresponding brightness temperatures in the umbra $(u)$ and the penumbra (p). The dashed lines denote a best-fit line to the data points corresponding to the umbra and penumbra.
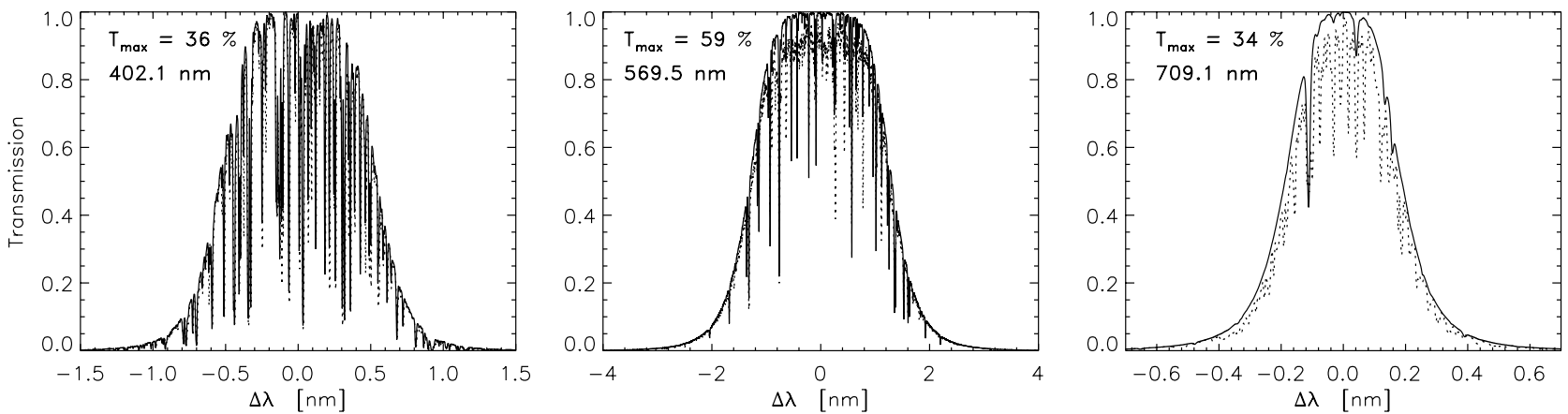

Fig. 7. The photospheric (full line) and umbral (dotted line) atlas profiles for the observed three wavelength ranges. Each spectrum is folded with the filter profiles. $T_{\max }$ : maximal transmission of the interference filter.

brightness averages are for the umbra $<3.5 \%$ and for the penumbra $<1 \%$. The darkest umbral regions are on average $\overline{I_{\text {min }}^{\mathrm{u}}}=0.27 I^{\odot}(4600 \mathrm{~K})$ bright with a minimum of $0.24 I^{\odot}(4510 \mathrm{~K})$.

Figure 5 shows typical temperature maps after the reconstruction and Fig. 6 illustrates the correlation of the brightness temperatures inside the umbra and the penumbra. The dashed line indicates a best-fit line to the entire set of points. In each of the three panels the points lie in a (narrow) band above the diagonal as a result of the general wavelength dependence of the brightness temperatures. The slope of the linear fits is slightly lower than that of the diagonal. Part of this deviation may come from changes in the temperature gradient in the continuum formation region: an anti-correlation of the temperature and its gradient causes a slightly lower slope of the fits. From 

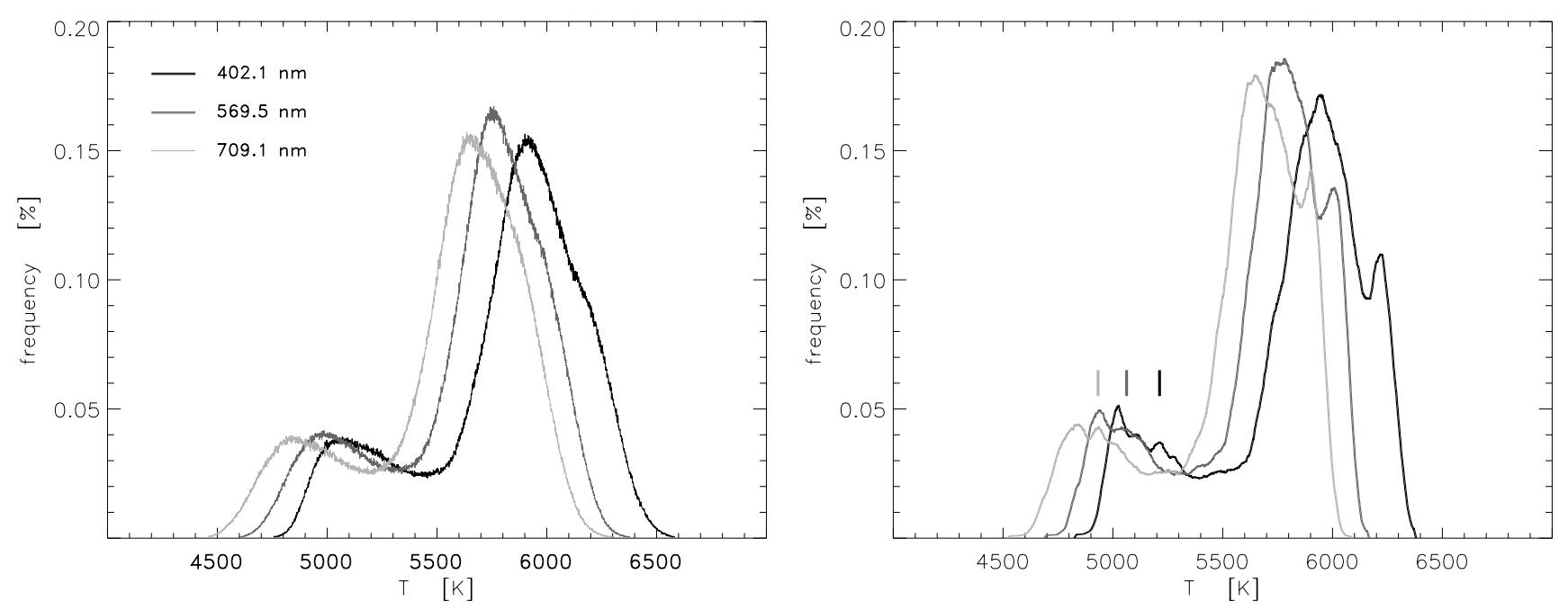

Fig. 8. Left: temperature histograms for the observed continua based on the whole sequence of temperature maps (71 frames). Right: temperature histograms based on the average temperature maps. The second components in the umbral temperature range are marked by short vertical lines. The maxima of the second component are approximately at $4930 \mathrm{~K}(402.1 \mathrm{~nm}), 5060 \mathrm{~K}$ $(569.5 \mathrm{~nm})$ and $5210 \mathrm{~K}(709.1 \mathrm{~nm})$.

Table 2. Radiation deficit in the undisturbed sun and a sunspot umbra in the three observed wavelength bands.

\begin{tabular}{|c|cc|}
\hline$\lambda$ & \multicolumn{2}{|c|}{ Radiation deficit $[\%]$} \\
\cline { 2 - 3 }$[\mathrm{nm}]$ & Quiet Sun & Umbra \\
\hline 402.1 & 22 & 28 \\
569.5 & 6 & 15 \\
702.1 & 3 & 19 \\
\hline
\end{tabular}

the current data it is, however, not possible to conclude whether the temperature and its gradient are correlated or anti-correlated, because the dominant contribution to the deviation of the slope comes from the strong temperature and wavelength dependence of the number and strength of absorption lines. As an illustration, Fig. 7 shows the photospheric (Kurucz et al. 1984) and umbral atlas profiles $(569.5 \mathrm{~nm}$ : Wöhl et al. 1970; $709.1 \mathrm{~nm}$ : Wallace et al. 1998) for the three wavelength ranges that we observed. For each of the spectra, folded with our filter profiles, we compute the radiation deficit caused by the presence of spectral lines in the window (Table 2).

This demonstrates that the temperature dependence of the radiation deficit is largest in the red, whereas the radiation deficit itself is largest in the blue. This causes brightness temperatures in the red, and to a lesser extent in the green, to decrease faster than the brightness temperatures in the blue when going towards cooler parts of the spot.

The distribution of temperature based on the whole sequence of 71 temperature maps (left side) and based on the time average (right side) is displayed in Fig. 8. The normalization refers to the total number of pixels, meaning $71 \times 281 \times 281$ for the total distribution and $281 \times 281$ for the distribution of average temperatures.
The total temperature histograms result from adding up the individual distribution functions and thus reflect the evolution in time and the noise in each resolution element. The distributions show two maxima due to typical or characteristic temperatures inside the penumbral $(5760 \mathrm{~K})$ and umbral regions $(4990 \mathrm{~K})$. None of the functions indicates a double-peaked distribution in the penumbral regions that could be ascribed to the filamentary structure of the penumbra. On the high-temperature side of the penumbral peak, all distributions show an asymmetry due to the spot surroundings. Because of its low area fraction the granulation contributes only little to the distribution function.

In contrast, the average distribution functions reveal much more fine structure: granular temperatures are well separated and show a third peak around $5990 \mathrm{~K}$. The penumbral component is slightly asymmetric towards higher temperatures, an effect that is less pronounced in the blue continuum but clearly visible with increasing wavelength. Inside the umbral range, the temperature distribution suggests the existence of more than one component (marked by the vertical bars). Characteristic temperatures inside umbra, penumbra and granulation are nearly identical to those of the summed temperature distributions. We want to remark that averaging over the $90 \mathrm{~min}$ observation period does not change the morphological appearance of the sunspot: neither the umbra nor the penumbra become homogenized. The filamentary penumbral structure is well-preserved, dark and bright filaments are clearly distinguishable, even though the comet-like shape of the bright filaments is lost. The umbra shows up very inhomogeneous and pervaded by reticular bright lanes, populated with umbral dots. The relevant intensities and corresponding brightness temperatures are summarized in Table 3 at the end of Sect. 9 

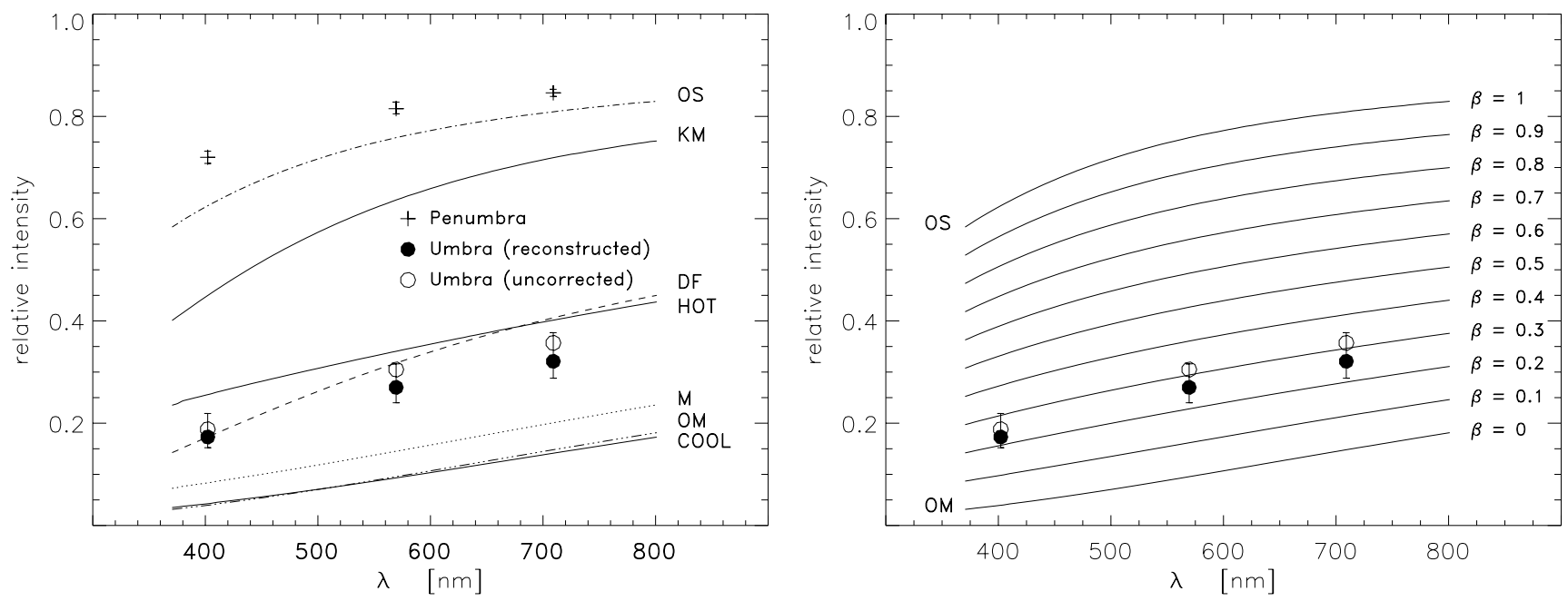

Fig. 9. Synthetic and observed relative intensities of umbra/quiet sun and penumbra/quiet sun. Left: comparison with onecomponent models. Right: comparison with a set of models that result from different mixtures of the hot (OS) and cold component (OM) of the two-component model of Obridko \& Staude (1988). $\beta$ : filling factor. KM: Penumbra, Kjeldseth Moe \& Maltby (1969). DF: Penumbra, Ding \& Fang (1989). OS, OM: Umbra (hot/cold), Obridko \& Staude (1988). HOT, COOL: Umbra, Collados et al. (1994). M: Umbra (model L), Maltby et al. (1986).

\subsection{Synthetic intensities}

The left side of Fig. 9 illustrates the spectral distribution of the observed averaged umbral and penumbral intensities, $\overline{I_{\min }^{\mathrm{u}}}$ and $\overline{\left\langle I^{\mathrm{p}}\right\rangle}$ respectively. For comparison, the results of the model calculations are displayed in the form of normalized intensities (with respect to the quiet sun model QS). The observed and synthetic umbral intensities differ significantly at least by a factor of 2 from each other. Only the model HOT leads to slightly higher intensities.

The result for the penumbra is also quite unsatisfactory. The observed penumbral intensities $(0.72,0.81,0.85)$ are higher by a factor of $2-4$. However, the penumbral intensities are comparable to those of the 2nd component of the umbral model of Obridko \& Staude $(0.63,0.76,0.81)$.

Since none of the models reproduces the observed intensities, an attempt was made to match the umbra observation by different mixtures of the hot and cold component of the Obridko \& Staude models OS and OM. Variation of the filling factor $\beta$ generates a set of spectral intensity curves that can be compared with the observation. The result is shown on the right side of Fig. 9. The comparison with the mixed models indicates, that, inside the umbra, the bright and hot component must occupy a fraction of $\beta=20-30 \%$ in order to fit the observation. A value of $\beta=25 \%$ leads to brightness temperatures of $4940 \mathrm{~K}$, $4605 \mathrm{~K}$ and $4460 \mathrm{~K}$ in the three observed continua. Except for the blue continuum $(402.1 \mathrm{~nm})$ this compares quite well to the observed temperatures $\overline{T_{\min }^{\mathrm{u}}}$ of $4790 \pm 50 \mathrm{~K}$, $4600 \pm 50 \mathrm{~K}$ and $4460 \pm 40 \mathrm{~K}$.

\section{Discussion and conclusions}

\subsection{Spectral distribution of sunspot intensities}

Figure 10 displays the wavelength dependence of observed umbral intensities. Additionally, the spectral distribution of umbral intensities derived from the Maltby model L (M) of an umbra in the late (middle) phase of the solar cycle is plotted. The observed intensities and temperatures are significantly higher than most existing observational results (Albregtsen \& Maltby 1981a,b; Albregtsen et al. 1984; van Ballegooijen 1984; Sobotka 1988; Bumba et al. 1990). Exceptions are the results obtained by Sütterlin \& Wiehr (1998) based on a speckle-reconstructed 3-colour photometry. Sütterlin \& Wiehr find brightness temperatures for the darkest umbral regions which are considerably higher than those of models for the darkest umbral cores (Kollatschny et al. 1980). The sunspot they observed is rather small and strongly inhomogeneous and therefore comparable to our specimen. As already mentioned in Sect. 8.2, only the umbral model HOT of a small sunspot (Collados et al. 1994) yields brightness temperatures up to $300 \mathrm{~K}$ higher $(5090 \mathrm{~K}, 4780 \mathrm{~K}, 4660 \mathrm{~K})$ than our observations in the three continuum bands.

As an obvious explanation for the discrepancy between our values and previous ones, an insufficient correction for photospheric stray light comes into question. The observed sunspot has a radius about 5 arcsec. For a limb distance of 5 arcsec the scattered intensity in the green continuum is $\sim 3 \%$. This corresponds to a lower limit of stray light since the estimation does not include the fact that light from all sides is scattered inside the umbra and not only from one side. Moreover, the position of minimum intensity is not located in the center of the umbra but rather $2-3$ arcsec near to the penumbral boundary. Towards smaller limb distances, the scattered intensity rises steeply. At a limb 


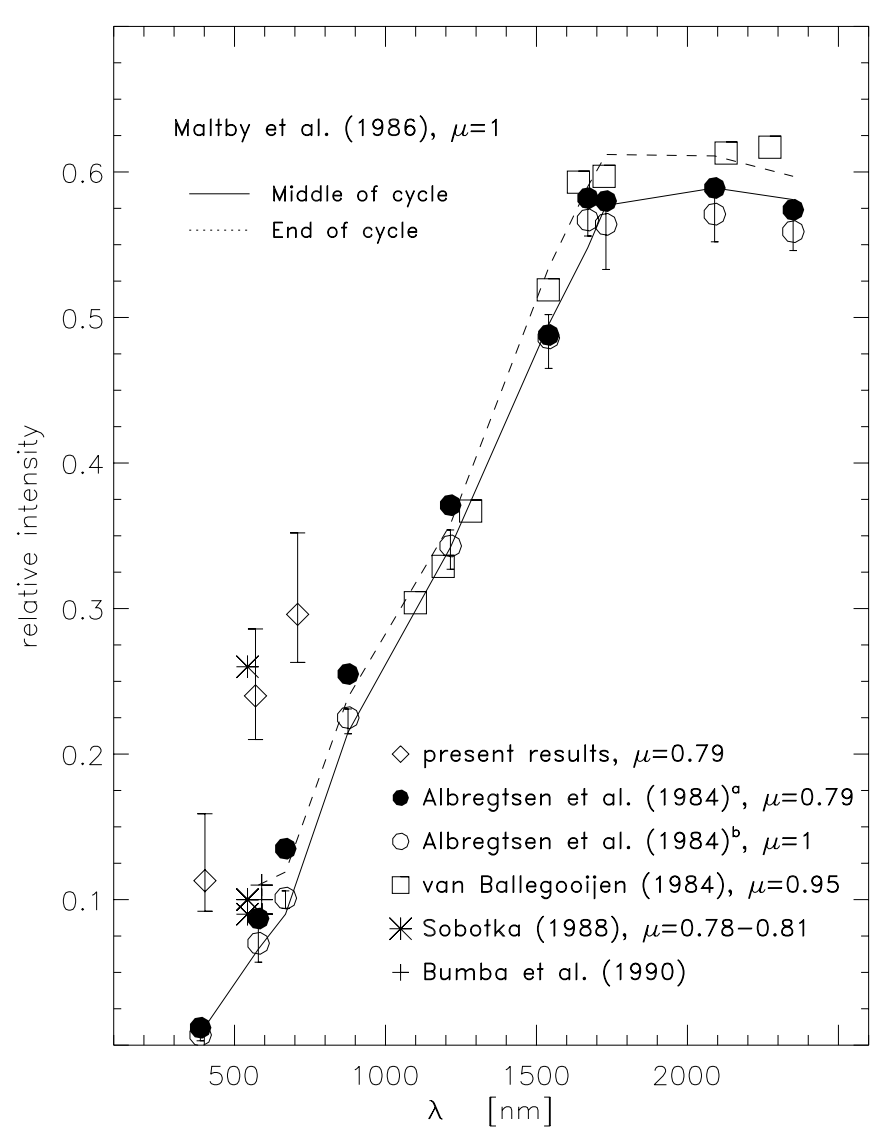

Fig. 10. Spectral distribution of the mean umbral continuum intensity $\overline{I_{\mathrm{min}}^{\mathrm{u}}}$ (stray light corrected). The bars indicate the range of observed intensities.

distance of 3 arcsec the amount of scattered light amounts to $5 \%$ and at 2 arcsec it is already $9.5 \%$. Under the assumption that the real contribution of stray light lies in this range, the observed umbral intensities are still higher, but within a few percent comparable with previous results.

However, from an observational point of view, a relation between brightness and sunspot size cannot be excluded, although the investigation of a systematic increase of umbral brightness with decreasing spot size is considerably complicated because a proper stray light correction for small spots is more difficult and unsecure. Therefore the umbral brightness of small spots and its wavelength dependence is almost unknown (Albregtsen \& Maltby 1981b). Existing results refer to large sunspots $\left(r_{\mathrm{u}}>\right.$ 5 arcsec) and reveal no significant dependence between umbral core brightness and sunspot size (Zwaan 1965; Rossbach \& Schröter 1970; Mykland 1973; Albregtsen \& Maltby 1981a; Albregtsen et al. 1984). Instead, it appears that the umbral core brightness varies with other parameters, like the phase of the solar cycle and the sunspot position (Albregtsen \& Maltby 1978; Albregtsen \& Maltby 1981a; Albregtsen et al. 1984). The conjecture that a different filling with UDs can account for the variation of umbral core brightness from spot to spot is not supported by Sobotka et al. (1993), where the mean umbral background intensity determines the umbral core brightness and not the individual filling with UDs.

Alternatively, more recent observations give increasing evidence for real differences in the thermal properties between the umbrae of large and small sunspots: smaller sunspots show brighter umbrae than larger ones (Sobotka 1988; Stellmacher \& Wiehr 1988; Kopp \& Rabin 1992; Solanki et al. 1992; Rüedi et al. 1992; Martínez Pillet \& Vázquez 1993; Sobotka et al. 1993; Collados et al. 1994). In particular the results obtained by Kopp \& Rabin (1992), Solanki et al. (1992) and Rüedi et al. (1995) point towards a linear dependence between the minimum umbral brightness and the sunspot diameter in the observed spectral range at $1.56 \mu \mathrm{m}$. These results are supported by Martínez Pillet \& Vázquez (1993), who investigated Stokes- $V$ profiles of three spectral lines in the visible with different excitation potentials. Further, Collados et al. (1994) deduce atmospheric models including the variation of the magnetic field strength and the line-of-sight velocity with height for a large and a small sunspot. The umbral atmosphere of the small sunspot turns out to be hotter, with a smaller magnetic field strength at $\tau=1$ and above.

A link between umbral brightness and sunspot size can be drawn also from the thermal-magnetic relation (Kopp \& Rabin 1992; Martínez Pillet \& Vázquez 1993; Solanki et al. 1993; Balthasar \& Schmidt 1993; Rüedi et al. 1995), meaning the local or overall relationship between the sunspots magnetic field strength and temperature. Common sense expects, that the minimum umbral temperature differs from sunspot to sunspot but coincides with the location of maximum field strength and both quantities appear to be linear functions of the sunspot size.

Our observed umbral core brightness and temperature confirms these recent findings and is further supported by the observed mean penumbral brightness, $\overline{\left\langle I^{\mathrm{p}}\right\rangle}$, shown in Fig. 11 and compared to previous results. Our values deviate on average by $8 \%$ from the results of Maltby (1972), based on the photographic observation of several sunspots. Their data is stray light corrected but suffers from a low spatial resolution. The spectral distribution of the mean penumbral brightness obtained by Sütterlin \& Wiehr (1998) deviates in particular for their blue and red continuum from previous observations. In our green and red continuum the observed values lie well inside the range given by Grossmann-Doerth \& Schmidt (1981). The authors distinguish between quiet and active parts of the penumbra. Their values are not stray light corrected, but treated with a Fourier technique in order to minimize atmospheric and instrumental influence.

Because of the spatial averaging and the smaller intensity difference between penumbra and quiet sun, the presence of stray light is of less impact on the intensity $\left.<I^{\mathrm{p}}\right\rangle$, as compared to $I_{\text {min }}^{\mathrm{u}}$. The error in the penumbral brightness due to stray light is likely to be in the range of $1 \%$. In addition, the brightness of the darkest part of the umbra appears to be well correlated with the penumbral 
Table 3. Intensities (upper row) and the corresponding brightness temperatures (lower row) inside the umbra and penumbra. $\left(I_{\text {min }}^{\mathrm{u}}\right)_{-}, \overline{I_{\text {min }}^{\mathrm{u}}}$ : minimum umbral intensity and its average in time. $\widehat{I}^{\mathrm{u}}, \widehat{I}^{\mathrm{p}}$ : characteristic umbral and penumbral intensity taken from the total distribution function (all 71 frames). $\overline{\left\langle I^{\mathrm{p}}\right\rangle}$ : average spatial penumbral brightness $\overline{C_{\mathrm{rms}}^{\mathrm{p}}}$ : average rms-intensity fluctuations of the penumbra.

\begin{tabular}{|c|ccc|ccc|}
\hline \multirow{2}{*}{$\lambda[\mathrm{nm}]$} & \multicolumn{3}{|c|}{ Umbra } & \multicolumn{3}{c|}{ Penumbra } \\
\cline { 2 - 7 } & $\left(I_{\mathrm{min}}^{\mathrm{u}}\right)_{-}$ & $\overline{I_{\min }^{\mathrm{u}}}$ & $\widehat{I}^{\mathrm{u}}$ & $\overline{\left\langle I^{\mathrm{p}}\right\rangle}$ & $\overline{C_{\mathrm{rms}}^{\mathrm{p}}}$ & $\widehat{I}^{\mathrm{p}}$ \\
\hline \multirow{3}{*}{402.1} & 0.15 & $0.17 \pm 0.01$ & 0.23 & $0.720 \pm 0.005$ & $11.3 \pm 0.9$ & 0.68 \\
& 4710 & $4790 \pm 50$ & 5050 & $5910 \pm 10$ & $150 \pm 10$ & 5880 \\
\hline \multirow{2}{*}{569.5} & 0.24 & $0.27 \pm 0.01$ & 0.39 & $0.81 \pm 0.004$ & $9.5 \pm 0.7$ & 0.79 \\
& 4510 & $4600 \pm 50$ & 4940 & $5750 \pm 10$ & $150 \pm 10$ & 5740 \\
\hline \multirow{2}{*}{709.1} & 0.29 & $0.32 \pm 0.01$ & 0.44 & $0.85 \pm 0.003$ & $8.8 \pm 0.8$ & 0.82 \\
& 4360 & $4460 \pm 40$ & 4820 & $5640 \pm 10$ & $160 \pm 10$ & 5630 \\
\hline
\end{tabular}

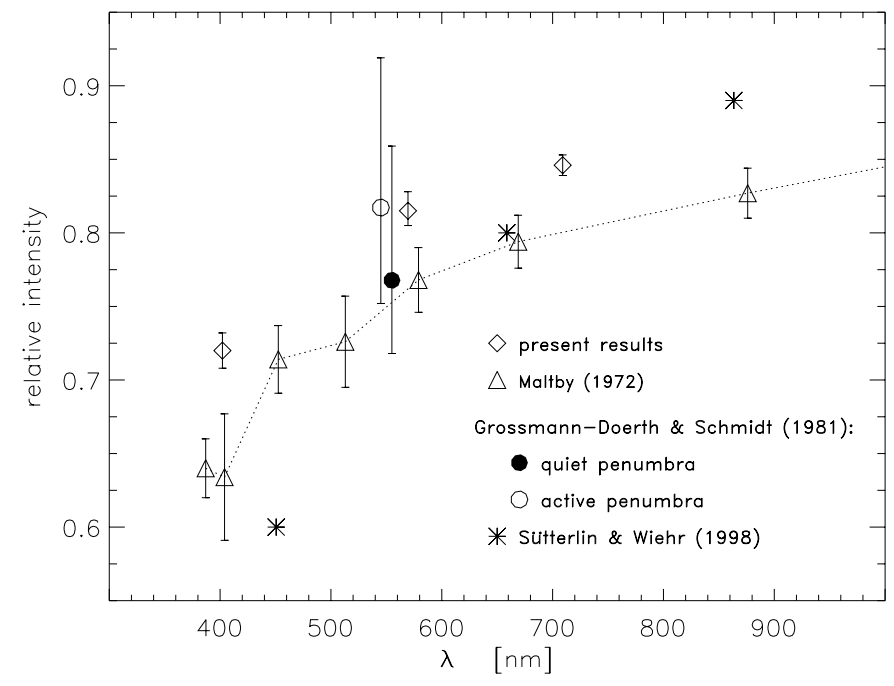

Fig. 11. Spectral distribution of the spatially averaged penumbral continuum intensity $\overline{\left\langle I^{\mathrm{p}}\right\rangle}$. The bars indicate the range of observed intensities.

brightness (Ekman 1974). We therefore consider the difference in the mean penumbral brightness and the umbral core brightness between our results and previous results mostly as real and conclude that the observed umbral core brightness and the related enhanced radiation flux points towards a more efficient transport of energy in the observable (and immediate subphotospheric?) layers of the umbral atmosphere of a small sunspot.

Theoretically, the strongest argument for a relation between brightness and size of sunspots was first proposed by Biermann (1941): the heat deficit and the lowered temperature in sunspots is due to a mechanism that is more efficient the stronger the magnetic fields are. In the strong vertical magnetic field, horizontal convective motions are inhibited, on the assumption that the concept of frozen fields (Alfvén 1942) applies. From the stronger magnetic field in large spots, it can be expected that large spots have a lower temperature than small spots. However, sunspot umbrae still carry approximately $20 \%$ of the photospheric radiation flux although the convection should be suppressed. Horizontal radiation-energy transport due to the temperature difference between the umbra and the surroundings can account only for $0.1 \%$ of the total flux and is thus negligible (Deinzer 1965). The question "what makes umbrae as bright as observed" is as fundamental as it is unclarified. The verification that small sunspots may exhibit different properties in the context of energy transport is therefore of great importance. There is a great need for a better statistics in the observation of small sunspots over a wide spectral range and with the highest possible spatial resolution which could be achieved with an adaptive optics system.

Acknowledgements. The authors thank the referee for carefully reading the manuscript and useful comments. We are very grateful to Jo Bruls, Helmold Schleicher and Oskar von der Lühe for their support and valuable discussions. Part of this work was supported by the German Deutsche Forschungsgemeinschaft, DFG.

\section{References}

Acton, D. S., Soltau, D., \& Schmidt, W. 1996, A\&A, 309, 661 Albregtsen, F., \& Maltby, P. 1981a, Sol. Phys., 74, 147

Albregtsen, F., \& Maltby, P. 1981b, in The physics of sunspots, Proceedings of the conference at the NSO Sac Peak Observatory, ed. L. E. Cram, \& J. H. Thomas, Sunspot NM, 127

Albregtsen, F., Jorås, P. B., \& Maltby, P. 1984, Sol. Phys., 90, 17

Alfvén, H. 1942, Arkiv. mat. astron. fys., 29b, 2

Allen 1955, in Astrophysical Quantities (The Athlone Press, University of London)

Ballegooijen van, A. A. 1984, Sol. Phys., 91, 195

Ballegooijen van, A. A., Nisenson, P., Noyes, R. W., et al. 1998, ApJ, 509, 435

Balthasar, H., \& Schmidt, W. 1993, A\&A, 279, 243

Berger, T. E., Löfdahl, M. G., Shine, R. S., \& Title, A. M. 1998a, ApJ, 495, 973

Berger, T. E., Löfdahl, M. G., Shine, R. S., \& Title, A. M. 1998b, ApJ, 506, 439 
Biermann, L. 1941, Vierteljahrschrift der Astronomischen Gesellschaft, 76, 194

Bumba, V., Sobotka, M., \& Simberová, S. 1990, in The dynamic sun, Publ. Debrecen Heliophys. Obs. 7, ed. L. Desz $\varnothing$, 84

Chandrasekhar, S. 1961, in Hydrodynamic and Hydromagnetic Stability (Oxford University Press, Oxford)

Collados, M., Martínez Pillet, V., Ruiz Cobo, B., del Toro Iniesta, J. C., \& Vázquez, M. 1994, A\&A, 291, 622

Deinzer, W. 1965, ApJ, 141, 548

Ding, M. D., \& Fang, C. 1989, A\&A, 225, 204

Druesne, P., Borgnino, J., Martin, F., Ricort, G., \& Aime, C. 1989, A\&A, 217, 229

Ekmann, G. 1974, Sol. Phys., 38, 73

Gonsalves, A. R. 1982, Opt. Eng., 21, 829

Grossmann-Doerth, U., \& Schmidt, W. 1981, A\&A, 95, 366

Hirzberger, J., Koschinsky, M., Kneer, F., \& Ritter, C. 2001, A\&A, 367, 1011

Holweger, A., \& Müller, E. A. 1974, Sol. Phys., 39, 19

Jahn, K., \& Schmidt, H. U. 1994, A\&A, 290, 295

Keller, C. U., \& Koutchmy, S. 1991, ApJ, 379, 751

Kentischer, T. 1995, A\&AS, 109, 553

Kjeldseth Moe, O., \& Maltby, P. 1969, Sol. Phys., 8, 275

Kollatschny, W., Stellmacher, G., Wiehr, E., \& Falipou, M. A. 1980, A\&A, 86, 245

Kopp, G., \& Rabin, D. 1992, Sol. Phys., 141, 253

Koschinsky, M., Kneer, F., \& Hirzberger, J. 2001, A\&A, 365, 588

Krieg, J., Kneer, F., Koschinsky, M., \& Ritter, C. 2000, A\&A, 360,1157

Kurucz, R. L., Furenlid, I., Brault, J., \& Testerman, L. 1984, Solar Flux Atlas from $296 \mathrm{~nm}$ to $1300 \mathrm{~nm}$, Kitt Peak NSO

Löfdahl, M. G., \& Scharmer, G. B. 1994, A\&AS, 107, 243

Löfdahl, M. G. 1996, Dissertation, Stockholm

Lühe von der, O. 1993, A\&A, 268, 374

Maltby, P. 1972, Sol. Phys., 26, 76

Maltby, P., Avrett, E. H., Carlsson, M., Kjeldseth-Moe, O., Kurucz, R. L., \& Loeser, R. 1986, ApJ, 306, 284

Maltby, P. 1992, in Sunspots: Theory and Observations, ed. J. H. Thomas, \& N. O. Weiss (Kluwer), 103
Martínez Pillet, V., \& Vázquez, M. 1993, A\&A, 270, 494

Mykland, N. 1973, Sol. Phys., 28, 49

Neckel, H. 1996, Sol. Phys., 167, 9

Neckel, H., \& Labs, D. 1984, Sol. Phys., 90, 205

Noll, R. J. 1976, J. Opt. Soc. Am., 66, 207

Obridko, V. N., \& Staude, J. 1988, A\&A, 189, 232

Paxman, R. G., \& Seldin, J. H. 1992, in Real Time and PostFacto Solar Image Correction, Proceedings of the 13th NSO/Sacramento Peak Summer Workshop, 112

Paxman, R. G., Seldin, J. H., Löfdahl, M. G., Scharmer, G. B., \& Keller, C. U. 1996, ApJ, 466, 1087

Rossbach, M., \& Schröter, E.-H. 1970, Sol. Phys., 12, 95

Rüedi, I., Solanki, S. K., \& Livingston, W. 1995, A\&A, 302, 543

Schmidt, W., Großmann-Doerth, U., \& Schröter, E.-H. 1988, A\&A, 197, 306

Sobotka, M. 1988, Bull. Astr. Inst. Czechosl., 39, 236

Solanki, S. K. 1997, in Advances in the Physics of Sunspots, ed. B. Schmieder, J. C. del Toro Iniesta, \& M. Vázquez, ASP Conf. Ser., 118, 178

Solanki, S. K., Rüedi, I., \& Livingston, W. 1992, A\&A, 263, 339

Solanki, S. K., Walter, U., \& Livingston, W. 1993, A\&A, 277, 639

Sütterlin, P., \& Wiehr, E. 1998, A\&A, 336, 367

Title, A. M., Tarbell, T. D., \& Topka, K. P. 1987, ApJ, 317, 892

Title, A. M., Topka, K. P., Tarbell, T. D., et al. 1992, ApJ, 393,782

Wallace, L., Livingston, W. C., Bernath, P. F., \& Ram, R. S. 1998, An Atlas of the Sunspot Umbral Spectrum in the Red and Infrared from 8900 to $15.050 \mathrm{~cm}^{-1}$ (6642 to $11230 \AA$ ), Kitt Peak NSO

Wilken, V., de Boer, C. R., Denker, C., \& Kneer, F. 1997, A\&A, 325, 819

Wöhl, H., Wittmann, A., \& Schröter, E. H. 1970, Sol. Phys., 13, 104

Zwaan, C. 1965, in Sunspot Models: A Study of Sunspot Spectra, Rech. astr. Obs. Utrecht 17 part 4 (Dordrecht), The Netherlands 